\title{
Gehirn und Geist am Computer trainieren?
}

Zusammenfassung: Immer wieder findet man die Behauptung, dass Videound Computerspiele dazu geeignet seien, das Gehirn bzw. geistige Leistungen zu trainieren. Hierzu wird eigens programmierte Software verkauft oder es werden käufliche Spiele, die nicht zum Training programmiert sind, auf entsprechende Auswirkungen untersucht. Die Ergebnisse zeigen, dass zwar die jeweils bearbeitete Aufgabe besser/ schneller bearbeitet wird, diese Fähigkeit jedoch nicht auf andere Fähigkeiten generalisiert. Die vermeintlichen positiven Auswirkungen von (zu Unterhaltungszwecken produzierten) Computerspielen halten einer genaueren Betrachtung nicht stand und entpuppen sich u. a. als Fehlinterpretationen der (zu erwartenden) höheren Ablenkbarkeit. Die Möglichkeiten der modernen Gehirnforschung wurden verwendet, um die Auswirkungen bei der vermeintlicher „Trainingsverfahren“ (Trainingssoftware und Unterhaltungssoftware) auf die Struktur und die Funktion des Gehirns zu untersuchen. Wieder werden Befunde fehlinterpretiert und aus einer klar erkennbaren Beeinträchtigung der normalen Gehirnentwicklung als Anzeichen einer vermeintlichen Verbesserung gewertet. Schließlich werden Aktivierungsmuster je nach Bedarf interpretiert, vermehrte Aktivierung als klares Zeichen von Verbesserung und verminderte Aktivierung als klares Zeichen von Aufwandsverminderung (also ebenfalls Verbesserung). Auf die bekannten Risiken und Nebenwirkungen wird in der Literatur zur vermeintlichen Verbesserung geistiger Leistungen durch Computerspiele praktisch nie hingewiesen, was man - wie die „positiven" Ergebnisse auch - als Zeichen des Einflusses massiver Lobbyarbeit einer milliardenschweren Spieleindustrie werten kann, die privatwirtschaftlich Gewinn produziert. Wissenschaftlich nachgewiesene positive Auswirkungen von Computerspielen existieren nicht, ihre negativen Folgen, deren Kosten die Allgemeinheit trägt, sind nachgewiesen.

Die Frage, ob man am Computer den Geist trainieren könne, wird bis heute kontrovers diskutiert. Zum einen gibt es Firmen wie Lumosity, BrainHQ, Cogmed oder CogniFit, die mit dem Verkauf von Gehirntrainingssoftware Millionen verdienen. Sie werben mit Sprüchen wie „improve your brain while having fun“, „cutting edge science personalized for you“ oder "will help you academically, socially, and professionally [by] allowing you to focus and resist distractions better" ${ }^{\text {[ }}$ 41]. Zugleich ist jedoch die Evidenz für die Wirksamkeit solcher „Gehirngymnastik" mit Computer, Bildschirm, Maus und Tastatur unter Wissenschaftlern umstritten.

Zum anderen gibt es die Computerspieleindustrie, die mit dem Verkauf von Computerspielen Milliarden verdient und immer wieder das fertig zu bringen scheint, was die Gehirngymnastik-Leute nicht hinbekommen: Studien, die vermeintlich klar zeigen, dass Computerspiele die geistige Leistungsfähigkeit verbessern. Im Gegensatz zur Gehirntrainingssoftware sind Computerspiele jedoch nicht eigens dafür programmiert, solcherlei Trainingseffekte zu produzieren, und sie werden auch nicht damit beworben. Diese eigenartige Situation bedarf der Erklärung, denn die Frage, ob geistige Leistungen am Computer trainierbar sind (und wenn ja, wie dies am besten geschehen sollte) hat erhebliche praktische, medizinische und ökonomische Relevanz. Wie also sieht die Datenlage wirklich aus?

1 Zu Deutsch etwa: „Verbessere dein Gehirn und hab Spaß dabei“, „Neueste wissenschaftliche Erkenntnisse für Sie personalisiert" oder „Wird Sie akademisch, sozial und beruflich voranbringen, [weil es Ihnen ermöglicht], sich auf das Wesentliche zu konzentrieren und Ablenkungen besser zu widerstehen".

\section{Gehirntrainingssoftware}

Die Firma Lumosity wurde im Jahr 2007 mit dem Ziel gegründet, bezahlte ${ }^{2}$ Online-Spiele zum Training von Aufmerksamkeit, Gedächtnis und Denken (Flexibilität, Geschwindigkeit und Problemlösefähigkeit) zur Verfügung zu stellen. Sie ist das größte und bekannteste Unternehmen, das adaptives kognitives Training kommerziell anbietet. Im Jahr 2015 hatte sie 23,6 Millionen US\$ Einnahmen von 70 Millionen zahlenden Mitgliedern (Wikipedia: Lumosity 2019). Eine Suche in der Medizindatenbank Pubmed zu „Lumosity [AND] cognitive training “ ergab 20 Treffer (Stand: 23.3.2019), die bei genauerer Betrachtung zumeist jedoch entweder das Design geplanter Studien oder Einzelfälle betrafen. Über positive Effekte berichtet eine im vergangenen Jahr publizierte Studie aus Saudi-Arabien an insgesamt 72 Teilnehmern im Alter von etwa 25 Jahren [1], die jedoch in einem unbekannten Journal publiziert wurde. Eine spanische Studie an 55 älteren Probanden (25 Teilnehmer in der Experimentalgruppe, 30 Teilnehmer in der Kontrollgruppe; mittleres Alter ca. 65 Jahre) ergab „bescheidene Effekte” des Gehirntrainings bei einigen der durchgeführten Tests [2]. Beide Studien sind wahrscheinlich aufgrund der geringen Teilnehmerzahlen (es wird über eine erhebliche Rate von Abbrechern berichtet) als eher „underpowered“ zu bezeichnen.

Die einzige bislang vorhandene gut publizierte kontrollierte randomisierte Studie zum Effekt der Trainingssoftware von Lumosity wurde im Fachblatt Journal of Neuroscience im August 2017 publiziert [20]. Den Autoren ging es nicht nur allgemein um das Training der geistigen Leistungsfähigkeit („cognitive performance“), sondern insbesondere um das Training von Selbstkontrolle („executive functions“), im

2 Die Preise lagen zwischen 14.95 US\$ für einen Monat und 299.95 US\$ für eine lebenslange Mitgliedschaft [52]. 
Sinne (1) von Risikoverhalten („risk sensitivity“) und (2) des Verzichts auf unmittelbare Belohnung zur Erlangung größerer Vorteile in der Zukunft („future discounting“). Der Grundgedanke war der, dass die Funktion des Arbeitsgedächtnisses, das im Frontalhirn, insbesondere im dorsolateralen präfrontalen Kortex (dIPFC) lokalisiert ist, im Verfolgen und Aufrechterhalten von längerfristigen Werten und Zielen besteht. Wäre diese Funktion trainierbar, so käme es zu weniger risikoreichen bzw. kurzfristig motivierten, oder gar impulsiven Entscheidungen und damit zu langfristig sinnvolleren Verhaltensweisen. Die Studie wurde an 128 Personen im Alter von 18 bis 35 Jahren durchgeführt (59\% hatten einen College-Abschluss, $44 \%$ weiblich, Durchschnittsalter 25 Jahre). Das Training lief über 10 Wochen und bestand in einer webbasierten computergestützten Anwendung (wöchentlich 5-mal für $30 \mathrm{Mi-}$ nuten) der Firma Lumosity oder von ähnlichen Kontrollspielen. Diese Kontrollspiele waren explizit so ausgewählt worden, dass sie 2 Eigenschaften nicht hatten: Sie waren weder adaptiv (d. h. ihr Schwierigkeitsniveau nahm während des Trainings nicht zu), noch waren sie eigens dafür programmiert, exekutive Funktionen zu trainieren. ${ }^{3}$ Gemessen wurden nicht nur die Auswirkungen des Trainings auf die trainierten Aufgaben, sondern auch die Generalisierung auf andere Aufgaben und nicht zuletzt Änderungen der Gehirnaktivität in bestimmten Bereichen des Frontalhirns mittels funktioneller Magnetresonanztomografie (fMRT).

Die Teilnehmer wurden aus 395 Probanden, die sich ursprünglich gemeldet hatten, mit Hilfe eines ausgeklügelten Verfahrens einzeln persönlich nach folgenden Aus-

3 Mit den Worten der Autoren: „The control training was designed to account not just for nonspecific placebo and social desirability effects, but also for two components believed to be critical to efficacy of adaptive cognitive training [...]. Unlike cognitive training, control games were not explicitly designed to tax executive functions and were not adaptive (i. e., difficulty levels were not adjusted over the course of training to users' current level of performance) [20]. schlusskriterien ${ }^{4}$ ausgewählt: Zu geringe Intelligenz $(n=10)$, bestehende Kontraindikationen für die Durchführung einer MRT ( $n=22$ ), zu hohe oder zu geringe Risikobereitschaft (außerhalb des Bereichs vom 5 . bis 95. Perzentil ( $n=52)$ bzw. zu hohe oder zu niedrige Diskontierung der Zukunft (außerhalb des Bereichs vom 10. bis 90. Perzentil ( $n=41$ ) oder beides ( $n=6)$ zur Vermeidung von Boden- oder Deckeneffekten sowie vorbestehende neurologische oder psychische Erkrankungen, Sucht (außer Nikotin), positiver Atemalkoholtest, Farbenblindheit, Linkshändigkeit und Klaustrophobie $(n=11)$. Ausgeschlossen wurde auch, wer zu diesem persönlichen Verfahren nicht erschienen ( $n=31)$ war. Die übrig gebliebenen hatten dann noch eine Woche eines Eingewöhnungsprogramms zu absolvieren (5-mal/Woche eine halbe Stunde Spiele der Kontrollbedingung spielen), um die Compliance, d. h. die Bereitschaft zum Mitmachen, zu überprüfen. Dies führte zum Ausschluss von weiteren 54 Probanden, weil sie nur 3 oder weniger Spiel-Sessions in der Woche absolvierten, ihren Aufgaben also nicht nachkamen. Die übrigen Probanden wurden in die Studie aufgenommen und der Randomisierung unterzogen. Im Verlauf der Studie brachen nochmals 20 Teilnehmer in der Trainingsgruppe und 18 Teilnehmer der Kontrollgruppe aus verschiedenen Gründen ab und kamen nicht zur Auswertung. Dies resultierte in einem Gesamt-N von 128, mit jeweils 64 Teilnehmern in der Trainings- und der Kontrollgruppe.

Für jede 30-minütige Trainingssitzung, die aus jeweils 10 bis 15 kleinen Trainingsspielen bestand, erhielten die Teilnehmer 5 US\$. Etwa die Hälfte der Teilnehmer absolvierten mehr als $70 \%$ aller 50 Trainingssitzungen („gute Mitmacher“), die andere Hälfte weniger als 70\% (,schlechte Mitmacher“). Die Intervention bestand aus Trainingsspielen für bestimmte kognitiven Domänen (d. h. Aspekten geistiger Leistungsfähigkeit) der Firma Lumosity. Diese

4 Dieses methodische Detail sei hier etwas genauer dargestellt, weil es die Schwierigkeiten beim experimentellen Arbeiten mit Probanden klar vor Augen führt: Man rekrutierte knapp 400 Probanden, um am Ende Daten von 128 Teilnehmern - also weniger als einem Drittel davon - auswerten zu können.
Trainingsspiele von 2 bis 3 Minuten Dauer wurden immer in der gleichen Reihenfolge von den Teilnehmern durchgeführt und betrafen die folgenden Domänen (prozentualer Anteil in Klammern): Arbeitsgedächtnis (27\%), Aufmerksamkeit (13\%), Flexibilität (24\%) Problemlösen (15\%) und Kurzzeitgedächtnis (12\%), und Verarbeitungsgeschwindigkeit ( $9 \%)$. Alles Trainingsspiele waren adaptiv, d. h. so konstruiert, dass sich ihr Schwierigkeitsgrad der Leistungsfähigkeit des Probanden ständig anpasste.

Die Teilnehmer in der Kontrollgruppe spielten in dieser Zeit ansprechende unterhaltsame Videospiele, die nicht zum Training programmiert waren und deren Schwierigkeitsgrad sich zwar im Laufe des Spiels ändern konnte, wobei diese Änderungen jedoch fest einprogrammiert (also nicht adaptiv) waren. Um Erwartungseffekte auszuschließen, lautete die Instruktion in beiden Gruppen gleich: „[...] participants in both groups were given the same information regarding the study purpose (e. g., "we are investigating the effects of certain types of computer games on brain activity and decision-making behavior," [20], S. 7392).

Vor und nach dem 10-wöchigen Training wurden standardisierte kognitive Testverfahren für Aufmerksamkeit (Continuous Performance Task), Arbeitsgedächtnis (visual/spatial n-back), Hemmung einer präpotenten Antwort (,response inhibition “: stop signal task), Interferenzanfälligkeit (Stroop test) und kognitive Flexibilität (color shape task) durchgeführt. Zudem wurde mittels funktioneller MRT die Gehirnaktivierung bei zwei experimentellen Paradigmen gemessen, bei denen es zum einen um Risikobereitschaft und zum anderen um die Diskontierung der Zukunft ging.

Die Ergebnisse der Studie waren sehr klar: Weder das spezifische, adaptive kognitive Training noch das Spielen der Spiele hatte einen Einfluss auf die Entscheidungsfähigkeit im Hinblick auf Risiko und Bewertung der Zukunft. Auch in den Tests zur Messung einzelner Indikatoren geistiger Leistungsfähigkeit gab es keine Unterschiede zwischen der Experimental- und der Kontrollgruppe. In beiden Gruppen gab es zwar geringe Fortschritte, die jedoch als Ergebnis aus dem Training der jeweiligen Aufgabe zu betrachten sind und nicht als Training 
einer allgemeinen geistigen Leistung. Und schließlich ergab auch die funktionelle Bildgebung keine differenziellen Resultate für das kognitive Training.

Die Autoren fassen ihre Ergebnisse wie folgt zusammen ([20], S. 7398): „Entgegen unseren Hypothesen fanden wir keinen Effekt des kognitiven Trainings auf die Gehirnaktivität bei der Entscheidungsfindung und ebenso keinen Effekt des kognitiven Trainings auf die Diskontierung der Zukunft und das Risikoverhalten. Beim Eingangstest („baseline“) fanden wir einen Zusammenhang zwischen Arbeitsgedächtnis und Diskontierung der Zukunft. Wenn die Effekte des kognitiven Trainings sich auf andere Aufgaben als die trainierten Aufgaben übertragen würden („transfer“), dann würde sich die Verbesserung der Maße für Arbeitsgedächtnis in Veränderungen der Zukunftsdiskonierung auswirken. " ${ }^{5}$ Unter beiden Bedingungen - dem kognitiven Training und den Spielen - kam es jedoch nicht zu solchen Transfereffekten und die Verbesserungen in den Tests waren zwischen den Gruppen nicht verschieden.

Die Autoren betonen, dass es nicht möglich ist, die Nullhypothese zu bestätigen, d. h. den Nachweis zu erbringen, dass kein Effekt besteht. Die Abwesenheit eines Beweises nicht das Gleiche ist wie ein Beweis der Abwesenheit (auf Englisch noch schöner, weil kürzer: absence of proof is not proof of absence). Dennoch heben die Autoren die Größe ihrer Stichprobe sowie ihr recht aufwändiges methodisches Vorgehen hervor. Mit anderen Worten: Wenn es einen Effekt gibt, dann kann er nicht besonders groß sein, denn ansonsten wäre er gefunden worden.

Aus genau diesem Grund ist auch eine im Jahr 2010 publizierte sehr große britische Studie von Interesse [31]. Die Autoren

5 "Contrary to our hypotheses, we found no effects of cognitive training on brain activity during decision-making and no effects of cognitive training on delay discounting or risk sensitivity. We did observe a baseline association between working memory and delay discounting. If the effects of cognitive training did transfer beyond the trained tasks, one would therefore expect that improvement on measures of working memory would result in changes in delay discounting." wandten sich an die Zuschauer der populärwissenschaftlichen englischen Fernsehserie Bang Goes The Theory, was sinngemäß übersetzt etwa bedeutet: „Mit einem Schlag ist wieder mal eine Theorie erledigt“. Die Zuschauer wurden gebeten, bei einer 6-wöchigen internetbasierten Studie mitzumachen. Daraufhin meldeten sich 52 617 Teilnehmer im Alter von 18 bis 60 Jahren, die zunächst mit 4 neuropsychologischen Tests im Hinblick auf logisches Denken, verbales Kurzzeitgedächtnis, räumliches Arbeitsgedächtnis und das Lernen paarweiser Wortassoziationen untersucht wurden. Diese Tests sind bekannt und sehr sensibel, sodass sie auch im klinischen Bereich als Tests auf krankhafte Beeinträchtigungen geistiger Leistungen eingesetzt werden. Sie zeigen aber auch Leistungsverbesserungen klar an, wenn es welche gibt.

Nach den Tests wurden die Teilnehmer nach dem Zufallsprinzip auf2 Experimentalgruppen und eine Kontrollgruppe verteilt. Sie mussten mindestens 3-mal wöchentlich für jeweils 10 Minuten 6 Trainingsaufgaben absolvieren, die in Experimentalgruppe I vor allem das logische Denken, Planen und Problemlösen betrafen. In Experimentalgruppe II wurde eine breitere Palette geistiger Leistungen mit Aufgaben zu Kurzzeitgedächtnis, Aufmerksamkeit, räumlichem Denken und Mathematik trainiert. Wie bei dem beschriebenen kommerziell erhältlichen Gehirntrainingsprogramm wurde die Schwierigkeit der Aufgaben dem jeweiligen Stand des Teilnehmers angepasst, so dass es immer neue Herausforderungen zu bewältigen gab und das Training nicht unter- oder überforderte. Die Kontrollgruppe erhielt keine Übungsaufgaben, sondern musste während des Trainings irgendwelche obskuren Fragen beantworten.

Nach 6 Wochen Training wurden die eingangs erhobenen Tests wiederholt und mit den Leistungen beim Eingangstest verglichen. 11430 Teilnehmer hatten durchgehalten und absolvierten den Eingangsund Ausgangstest sowie im Durchschnitt knapp 25 Trainingseinheiten. Gemessen wurden die Verbesserungen bei den 4 neuropsychologischen Tests (Leistung am Ende minus Leistung zu Beginn) sowie die Verbesserung bei den jeweils 6 im Training direkt bearbeiteten Aufgaben (ebenfalls Leistung am Ende minus Leistung zu
Beginn). Dieses Vorgehen erlaubte es, die trainingsspezifischen Verbesserungen von allgemeinen Verbesserungen der geistigen Leistungsfähigkeit zu unterscheiden. Mit anderen Worten: Es ließ sich ermitteln, ob die Teilnehmer das, was trainiert wurde, auch auf andere Situationen und Aufgabenübertragen konnten.

Die Ergebnisse der Studie waren klar und ernüchternd: Keines der Trainingsprogramme änderte etwas an der geistigen Leistungsfähigkeit der Teilnehmer. In allen 3 Gruppen kam es zu sehr geringen Verbesserungen in den Tests nach dem Training, die aber nicht auf das Training, sondern auf einen Übungseffekt beim Test zurückzuführen waren (man machte ja nach 6 Wochen noch einmal den gleichen Test). Im Hinblick auf logisches Denken, verbales Kurzzeitgedächtnis, räumliches Arbeitsgedächtnis und das Lernen neuer Gedankenverknüpfungen (Assoziationen) wurden keine Verbesserungen sichtbar. Demgegenüber verbesserten sich alle Teilnehmer der Experimentalgruppen I und II deutlich und überzufällig in den einzelnen Trainingsaufgaben. Selbst die Teilnehmer der Kontrollgruppe wurden ein wenig besser (statistisch aber nicht abgesichert) im Beantworten obskurer Fragen. Das bedeutet, dass das Training durchaus einen Lerneffekt hat, dieser Effekt einem jedoch bei anderen Aufgaben, selbst wenn sie mit dem Training verwandt sind, nichts nützt.

Die Autoren diskutieren ihre Daten daher auch sehr klar: „Unserer Ansicht nach liefern diese Ergebnisse keine Beweise für den weitverbreiteten Glauben, dass computerisierte Gehirntrainer bei gesunden Menschen die allgemeine geistige Leistungsfähigkeit verbessern“ ([31], S. 777, Übersetzung durch den Autor). ${ }^{6}$

Wem die Evidenz aus den genannten wissenschaftlichen Studien nicht ausreicht, der betrachte die juristischen Aspekte von Gehirntraining, das Trainingserfolge verspricht, die nicht nachweisbar sind. Aufgrund der im Jahr 2015 bestehenden Da-

6 „In our view these results provide no evidence to support the widely held belief that the regular use of computerized brain trainers improves general cognitive functioning in healthy participants beyond those tasks that are actually being trained.“ 
tenlage wurde nämlich der mit Lumosity betriebene Kommerz durch legale Sanktionen eingeschränkt. Am 5. Januar 2016 wurde Lumosity von der US-amerikanischen Verbraucherschutzbehörde Federal Trade Commission ${ }^{7}$ zu einer Strafzahlung von 2 Millionen US\$ wegen falscher Behauptungen in ihrer Produktwerbung verurteilt. „Lumosity nutzte die Ängste der Konsumenten im Hinblick auf die altersbedingte Abnahme ihrer geistigen Leistungsfähigkeit und behauptete, ihre Spiele könnten Gedächtnisschwund, Demenz und sogar die Alzheimer'sche Krankheit verhindern [...] Aber Lumosity konnte seine Werbung nicht wissenschaftlich untermauern“, schrieb die FTC (2016) in ihrer entsprechenden Verlautbarung. ${ }^{8}$

Wenn eigens zum Training programmierte Software auch nach den neuesten Metaanalysen [39] weder Gehirn noch Geist wirklich trainieren, dann sollte nicht eigens hierfür hergestellte Software erst recht keinen Effekt haben. Immer wieder wird jedoch behauptet, dass Computerspiele solche positiven Effekte haben.

\section{Computerspiele}

Im Koalitionsvertrag der gegenwärtigen Bundesregierung steht, dass E-Sport (und damit sind Computerspiele gemeint, nicht Fifa und ebenso wenig virtuelles Golfen oder Bogenschießen), ,wichtige Fähigkeiten schult, die nicht nur in der digitalen Welt von Bedeutung sind“ (Koalitionsvertrag 2018, S. 48, Zeilen 2168-2169). Auf-

7 Wörtlich übersetzt müsste man auf Deutsch von „Bundeshandelskommission“ sprechen. Es handelt sich um eine seit 1914 existierende unabhängige Bundesbehörde, die 1200 Mitarbeiter und ihren Sitz in Washington, DC, hat. „Sie wird bei direkten Beschwerden von Konsumenten oder Unternehmen gegen einzelne Unternehmen tätig. [...] Die Aufgabenstellung der Behörde wird allgemein damit beschrieben, unfairen und täuschenden Praktiken zu begegnen, um das Funktionieren eines konkurrenzbestimmten Marktes sicherzustellen“ [52].

8 “Lumosity preyed on consumers' fears about age-related cognitive decline, suggesting their games could stave off memory loss, dementia, and even Alzheimer's disease," said Jessica Rich, Director of the FTC's Bureau of Consumer Protection. "But Lumosity simply did not have the science to back up its ads" (FTC 2016; zit. nach [34]). grund dieser positiven Effekte wolle man darauf hinwirken, dass Computerspiele für gemeinnützig erklärt werden und damit steuerlich zu begünstigen sind.

Computerspiele werden von sehr vielen Menschen gespielt werden, in Deutschland von $42 \%$ aller Bürger bzw. von 81 \% der 14bis 29-Jährigen [3]. Zugleich sind nach den neuesten Daten hierzu 465000 Menschen von Computerspielsucht betroffen, einer mittlerweile von der Weltgesundheitsorganisation (WHO) als Krankheit anerkannten Form von Sucht. Zudem haben Computerspiele nachweislich negative Auswirkungen auf die Gesundheit, die Bildung und das Sozialverhalten eines Menschen [42].

Es gibt aber tatsächlich eine Reihe von Studien, die berichten, dass Computerspiele, bei denen außerirdische Wesen oder Terroristen mit den verschiedensten Waffen zu bekämpfen sind, die Reaktionsgeschwindigkeit oder sogar die Aufmerksamkeit trainieren können. Sehr oft wird dabei eine Arbeit aus dem Fachblatt Nature zitiert, das große mediale Interesse auf sich gezogen hatte und der zufolge das Spielen von Action Video Games die selektive Aufmerksamkeit verbessern soll [17]. Die Autoren der Studie behaupten, sie hätten anhand psychologischer Experimente nachgewiesen, dass Video-Game-Spieler („Gamer“) auf Ablenkreize besser reagieren können als Nicht-Video-Game-Spieler (Experiment 1). Auch können Gamer bei sehr rascher Abfolge von Reizen deren Anzahl vergleichsweise besser angeben (Experiment 2). Zudem reagieren Gamer stärker auf Ablenkreize, die sich weiter am Rande des visuellen Feldes befinden (Experiment 3) und weisen einen geringeren Attentional Blink Effect auf (Experiment 4). Wörtlich übersetzt bedeutet dies weniger „Aufmerksamkeitsblinzeln“, was dem mit Wahrnehmungspsychologie nicht vertrauten Leser wenig sagen dürfte. Daher sei kurz erläutert, worum es sich bei diesem Effekt handelt. Folgt etwa eine Fünftelsekunde (200 Millisekunden) nach der Darbietung eines Reizes ein zweiter Reiz, wird diesem zweiten Reiz normalerweise weniger Beachtung geschenkt, d. h. er wird in geringerem Ausmaß verarbeitet. Diese Verringerung fällt bei Gamern vergleichsweise geringer aus.
Die Experimente 1, 3 und 4 wurden mit jeweils 8 Gamern und acht Nicht-Video-Game-Spielern durchgeführt, bei Experiment 2 betrug die Gruppengröße jeweils 13. Die Gruppengrößen sind also bescheiden bis sehr bescheiden, aber dennoch wurde diese Arbeit zu einer Art Kronzeuge für die positiven Auswirkungen von Computerspielen auf die Spieler. Nach der Datenbank Pubmed wird sie beispielsweise von 289 weiteren wissenschaftlichen Publikationen zitiert (Daten abgerufen am 23.3.2019). Bei genauem Hinsehen belegen die in dieser Studie vorgelegten Daten jedoch keineswegs das, was sie zu belegen scheinen. Es ist vielmehr die sehr ungewöhnliche, von der Scientific Community deutlich abweichende Interpretation ihrer Daten, die zu diesem vermeintlichen Ergebnis führt. Im Einzelnen:

Da bei Ballerspielen ständig neue Gegner ins Sichtfeld treten, die bekämpft werden müssen, fand man bei den Gamern in Experiment 1 eine erhöhte Neigung zur Reaktion auf ablenkende Reize. Die wissenschaftliche Gemeinschaft der Aufmerksamkeitspsychologen ist sich weltweit einig darüber, dass dieser Befund im Sinne einer erhöhten Ablenkbarkeit - gleichbedeutend mit einer verminderten Aufmerksamkeit - zu interpretieren ist. Green und Bavelier diskutieren ihre Ergebnisse jedoch dahingehend, dass Computerspieler besser auf Ablenkreize reagieren und damit deren Aufmerksamkeit besser sei. Das ist etwa so, als würde man einen schnelleren Puls, eine höhere Körpertemperatur oder einen weiteren Bauchumfang als Zeichen von Gesundheit interpretieren, weil ja „schneller, höher, weiter“ grundsätzlich positiv konnotiert sei. Die Autoren verdrehten also einfach ein Defizit - vermehrte Ablenkbarkeit - ins angeblich Positive. Niemand sonst tut dies!

Nichts anderes verhalten sich die Dinge bei den weiteren 3 Experimenten zur selektiven Aufmerksamkeit. Wer auf viele Reize besonders schnell reagieren muss, kann sich auf einen Reiz schlechter konzentrieren (Ergebnis von Experiment 2). Wer beim Abschießen von Monstern aus dem All seine Aufmerksamkeit permanent überall auf den ganzen Bildschirm verteilen muss, der lernt dadurch, seine Aufmerksamkeit zu verteilen, und gerade nicht, sie zu fo- 
kussieren (Experiment 3). Bei selektiver Aufmerksamkeit geht es jedoch prinzipiell - das sagt schon der Name! - um deren Fokussierung. ${ }^{9}$

Um das Ergebnis des vierten Experiments zu verstehen, muss ich etwas ausholen: Die Verarbeitung eines Reizes in unserem Sehsystem braucht Zeit, auch wenn sie zunächst sehr rasch und automatisch erfolgt. So können wir beim Betrachten einer Szene während eines Zeitintervalls von nur 180 Millisekunden angeben, ob dabei ein Tier abgebildet ist oder nicht - also lange bevor wir auch nur bewusst richtig hingeschaut haben [48]. Damit wir überhaupt so schnell etwas wahrnehmen können, muss sich unser visuelles System gegen Überlastung schützen. Dies geschieht beim Attentional Blink, bei dem automatische Informationsverarbeitungsprozesse in unserem Sehsystem die Verarbeitung weiterer Reize stoppen, bis der nächste Reiz aufgenommen und verarbeitet werden kann. Dieser Vorgang dauert gut eine Viertelsekunde und ist kein Fehler des Systems, sondern ein wichtiger Schutzmechanismus vor Überlastung. Experiment 4 zeigt nun nichts weiter, als dass man sich diesen Mechanismus zumindest teilweise durch häufiges Video-Game-Spielen abtrainieren kann.

Schließlich wurden in einem fünften Experiment neun Nicht-Video-Game-Spieler über einen Zeitraum von 10 Tagen täglich jeweils eine Stunde lang mit einem EgoShooter-Spiel (Medal of Honor) trainiert. Eine Kontrollgruppe trainierte das gewaltfreie Spiel Tetris. „Dieses Spiel beinhaltet eine schwierige visuell-motorische Komponente, wohingegen Action Video Games eine Verteilung der Aufmerksamkeit bzw. einen häufigen Wechsel der Aufmerksamkeit erfordern. Bei Tetris muss man sich jeweils auf eine Sache konzentrieren, weswegen man bei diesem Spiel die beschriebe-

9 Und genau darum ging es in der Arbeit, die den Titel trägt: „Action video game modifies visual selective attention“. Die Autoren müssen gewusst haben, was sie da tun, denn sie hätten ja auch schreiben können, dass Action-Videospiele die selektive Aufmerksamkeit verbessern, was sie aber nicht taten! In ihrem Text reden sie zwar dauernd von Verbesserung, im Titel jedoch nur von „Modifizierung“, was sowohl Verbesserung als auch Verschlechterung bedeuten kann. nen Veränderungen der Aufmerksamkeit nicht erwarten würde“, schreiben die Autoren der Studie ([17], S. 537).

Die genannten Veränderungen der Aufmerksamkeit durch Ego-Shooter-Spiele wurden erneut gefunden, gehen aber in die falsche Richtung, denn sie zeigen, dass man sich dabei eine Aufmerksamkeitsstörung antrainiert! Noch einmal die Autoren: „Obgleich das Spielen von Video-Games ziemlich geistlos erscheint, kann es dennoch zu radikalen Veränderungen der visuellen Aufmerksamkeit führen“, fassen die Autoren Shawn Green und Daphne Bavelier ihre Ergebnisse zusammen ([17], S. 536). Sie suggerieren dem Leser damit positive Auswirkungen, die dann in den Medien auch in aller Ausführlichkeit breitgetreten wurden, ohne dass noch jemand nachgesehen, was wirklich herauskam. ${ }^{10}$ Fakt ist hingegen, dass diese Studie klar gezeigt hat, dass man mit Ego-Shooter-Spielen seine Konzentration und Selbstkontrolle abgibt, um sich wieder auf das mentale Funktionsniveau eines Reflexautomaten zu begeben. Es gibt also Veränderungen der Aufmerksamkeit - in Richtung einer Aufmerksamkeitsstörung! Und genau dies haben die Autoren nachgewiesen.

Die gleiche Arbeitsgruppe publizierte - teilweise ähnlich hochrangig [58] - weitere Studien zum Thema, die jedoch im Hinblick auf die Ergebnisse einerseits und die Art der Interpretation nicht anders wurden. So wurde bei 7- bis 22-jährigen Probanden erneut gefunden, dass Ablenkreize (flanker) bei Spielern von Video-Games „besser verarbeitet wurden“, d. h. mehr Ablenkung und weniger Fokussierung der Aufmerksamkeit. Die Autoren sprechen von einem höheren Maß an Aufmerksamkeit und „Spill-over-Effekten“ dieser vermeintlich vermehrten Aufmerksamkeit auf ablenkende Reize [57]. An einer einzigen Stelle der Diskussion ihrer Ergebnisse erwähnen sie, dass man dies auch anders sehen könnte: „Wir möchten anmerken, dass wir nicht eindeutig sagen können, ob ein größerer Testwert [...] tatsächlich durch erhöhte Aufmerksamkeitsressourcen oder

10 Wie es dazu kommen konnte, dass diese Studie in Nature publiziert wurde und auch von Fachleuten nie kritisch bewertet wurde, ist mir bis heute ein Rätsel. durch schlechte selektive Aufmerksamkeit zustande kommt“ ([57], Übersetzung durch den Autor). ${ }^{11}$ Aber nach dieser bemerkenswerten Einsicht wird gleich wieder weiter vernebelt. Eine ganze Reihe methodisch sehr aufwändiger und mit genügend statistischer Power versehenen (d. h. einer großen Zahl von Teilnehmern durchgeführten) Studien konnte keinerlei positive Auswirkungen von Computerspielen (welcher Art auch immer) auf die geistige Leistungsfähigkeit finden [29, 41, 49].

Selbst die oft behauptete größere Schnelligkeit (d. h. verminderte Reaktionszeiten) bei Video-Spielern wurde nicht bestätigt: Von 30 berechneten Korrelationen (des Video-Spiele-Spielens mit den Ergebnissen verschiedener Test geistiger Leistungsfähigkeit) waren nur 6 signifikant und numerisch klein. 3 dieser 6 Korrelationen waren positiv, die anderen 3 negativ, d. h. es gab in manchen Tests auch signifikant langsamere Reaktionen bei Gamern im Vergleich zu Nicht-Spielern. „Insgesamt deuten die vorliegenden Ergebnisse darauf hin, dass entweder keine oder nur sehr schwache Beziehungen zwischen dem Spielen unterschiedlicher Video-Spiele und grundlegenden geistigen Leistungen (Arbeitsgedächtnis, fluide Intelligenz, selektive Aufmerksamkeit und Verarbeitungsgeschwindigkeit) besteht", kommentieren abschließend die Autoren [49]. ${ }^{12}$

\section{Computerspiele im Scanner}

Nicht nur die auf der Verhaltensebene reklamierten „Verbesserungen“ psychischer Leistungen halten der Überprüfung nicht stand, sondern auch die Behauptungen, dass sogar mittels der modernen Neurowissenschaft gezeigt werden könne, dass Video-Spiele positive Auswirkungen auf das Gehirn hätten. Es wurden zwar immer wieder Studien publiziert, die dies zu belegen

11 Im Original: „We note that the ANT flanker compatibility score cannot unambiguously resolve whether a greater score is in fact due to enhanced attentional resources or due to poor attentional selection.“

12 „Overall, the current results suggest weak to nonexistent relations between video-game experience-across a variety of different games-and fundamental cognitive abilities (working memory, fluid intelligence, attention control, and speed of processing).“ 
scheinen. Wie schon bei den auf Verhaltensdaten beruhenden Studien ist jedoch auch in diesem Bereich die Interpretation der Ergebnisse zuweilen fragwürdig. Betrachten wir ein Beispiel:

Im Jahr 2014 publizierte das IMAGEN-Konsortium, ein Verbund von Bildgebungs-Wissenschaftlern bzw. Institutionen, eine große Studie an 152 Probanden zur Frage, ob Computerspiele einen Einfluss auf die Gehirnanatomie von 14-jährigen Jugendlichen haben [25]. Mittels struktureller MRT wurde gefunden, dass die mit dem Spielen von Computer-Spielen verbrachte Zeit (in Stunden pro Woche) mit der Dicke der Gehirnrinde insgesamt, und vor allem im Bereich des linken dorsolateralen präfrontalen Kortex (dIPFC) und der linken frontalen Augenfelder positiv korreliert ist. Hingegen fand man in keinem Bereich des Gehirns Hinweise auf eine Abnahme der Gehirnrinde in Abhängigkeit von der mit Computerspielen verbrachten Zeit. Die Autoren erklären diesen Befund als Bestätigung der vermeintlich bereits bekannten Befunde zu einer Verbesserung der kognitiven Leistungsfähigkeit durch das Spielen von Computerspielen. Um es mit ihren eigenen Worten zu sagen: „Der dIPFC ist das wesentliche Korrelat von exekutiver Kontrolle und strategischem Planen, welches wiederum die wesentlichen kognitiven Domänen für das erfolgreiche Spielen von Computerspielen sind. Die frontalen Augenfelder sind die Schlüsselregion für die Integration visueller und motorischer Signale und bedeutsam für Augenbewegungen und die Steuerung der visuell-räumlichen Aufmerksamkeit, also von Prozessen, die extensiv bei den Computerspielen involviert sind. Diese Ergebnisse könnten die biologische Basis der berichteten Verbesserungen geistiger Leistungsfähigkeit durch Computerspiele darstellen“ ([25], S. e1; Übersetzung durch den Autor). ${ }^{13}$

13 Im Original: „DLPFC is the core correlate of executive control and strategic planning which in turn are essential cognitive domains for successful video gaming. The FEFs are a key region involved in visuo-motor integration important for programming and execution of eye movements and allocation of visuo-spatial attention, processes engaged extensively in video games. The results may represent the biological basis of previously reported cognitive improvements due to video game play."

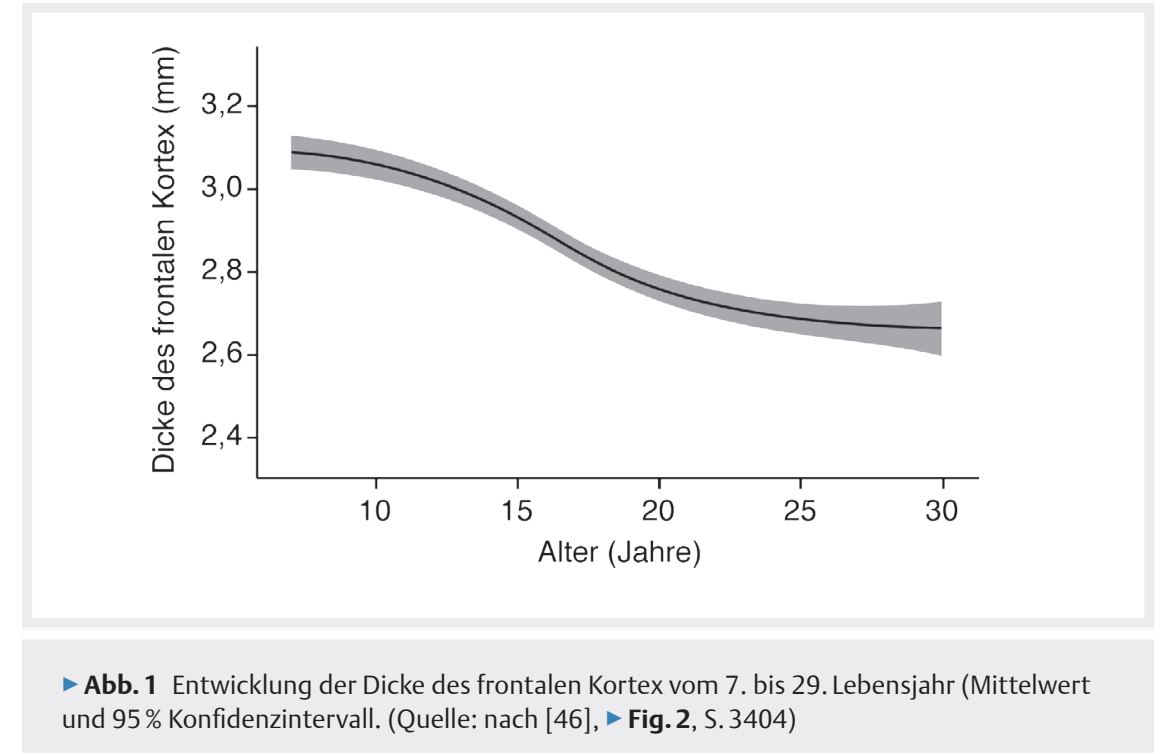

Interessanterweise kam niemand aus der großen Gruppe des IMAGEN-Konsortiums (die Arbeit hat 21 Autoren) auf die im Lichte der Erkenntnisse zur Gehirnentwicklung sehr naheliegende Idee, dass das Ergebnis auch genau umgekehrt interpretiert werden kann: Seit nunmehr 20 Jahren ist bekannt, dass die Dicke der Gehirnrinde im Laufe ihrer normalen Entwicklung in Kindheit und Jugend abnimmt [15]. Der Effekt wurde immer wieder bestätigt [13-16], mittlerweile auch an großen Datensätzen ([46]; > Abb. 1). Ein dickerer Kortex bei Gamern kann also auch im Sinne einer (noch) nicht erfolgten normalen kortikalen Abnahme interpretiert werden, also im Sinne einer Entwicklungsverzögerung.

Dafür spricht zudem, dass man bei einer Studie an 223 Kindern mit Aufmerksamkeits-Hyperaktivitätsstörung (ADHD) im Vergleich zu einer Kontrollgruppe von ebenfalls 223 Kindern gefunden hat: Der Kortex der Kinder mit ADHD war dicker, denn dessen Verdünnung als Zeichen seiner normalen Entwicklung hinkte im Vergleich zur Kontrollgruppe um 3 Jahre hinterher. Genauso diskutieren die Autoren auch - im Jahr 2007 - ihre Ergebnisse: „Die Entwicklungsverzögerung war in präfrontalen Regionen der Gehirnrinde am deutlichsten ausgeprägt, die für die Kontrolle kognitiver Prozesse, einschließlich Aufmerksamkeit und motorische Planung wichtig sind. Die Verzögerung der regionalen kortikalen Entwicklung bei ADHD wurde bislang nicht mit neuroanatomischen Methoden nachgewiesen“ ([40], S. 19659; Übersetzung durch den Autor). ${ }^{14}$ Dass es bei der Nutzung von Bildschirmmedien und vor allem bei Gamern zu Aufmerksamkeitsstörungen kommt, ist zudem aus einer ganzen Reihe von Studien bekannt [12, 23, 28, 35, 55].

Weiterhin wurde im Jahr 2013 bei 18 jungen Erwachsen (Durchschnittsalter 19 Jahre) mit Online-Spielsucht (online gaming addiction) im Vergleich zu Kontrollpersonen eine Verdickung bestimmter kortikaler Areale beschrieben [54]. Es gab also durchaus in der Literatur Befunde, die diese ganz andere - nämlich genau gegenteilige - Interpretation der Ergebnisse des Konsortiums deutlich nahegelegt hätten.

Auch nach der IMAGEN-Publikation hat sich die Befundlage hierzu noch weiter verbessert: Im Fachblatt Neuropsychologia erschien eine Studie mit dem treffenden Titel When less is more: Thinner fronto-parietal cortices are associated with better forward digit span performance during early childhood [5]. Bei Kindern im Alter von 4 bis 8 Jahren wurden das Arbeitsgedächtnis mit einem bekannten Test zum Zahlen nachsprechen gemessen und zugleich die Dicke des Kortex gemessen. Hierbei zeigte sich, dass ein dünnerer Kortex mit einer besseren Leistung des Arbeitsgedächtnisses korreliert ist. In einer weiteren Studie

14 „The delay was most prominent in prefrontal regions important for control of cognitive processes including attention and motor planning. Neuroanatomic documentation of a delay in regional cortical maturation in ADHD has not been previously reported.“ 
zeigte sich zudem auch für Bereiche des Hippocampus ein negativer Zusammenhang zwischen Volumen und Gedächtnisleistung: „in younger children bigger was better, whereas in older children smaller was superior" [36]. Eine Studie aus unserem Hause [45] an 103 jungen Menschen im Alter von 12 bis 27 Jahren, von denen 60 depressiv erkrankt waren, ergab ebenfalls eine Entwicklungsverzögerung im Sinn eines größeren Volumens der grauen Substanz vor allem im dIPFC bei den depressiven Patienten. Ob es sich hierbei um einen Risikofaktor oder um eine Folge der Erkrankung handelt, ist bislang nicht geklärt. Zusammenfassend lässt sich also sagen, dass die Behauptung, die Neurowissenschaft habe mittlerweile gezeigt, dass Videospiele dem Gehirn guttun, definitiv unzutreffend ist. Eine dickere Gehirnrinde im Kindes- und Jungendalter ist Ausdruck einer Entwicklungsverzögerung und einer geringeren Leistung des Arbeitsgedächtnisses und steht im Zusammenhang mit Suchtverhalten und Störungen der Aufmerksamkeit.

Die Erstautorin der IMAGEN-Studie publizierte im gleichen Jahr eine weitere Studie, bei der junge Erwachsene (Durchschnittsalter $24 \pm 3$ Jahre) über 2 Monate hinweg täglich 30 Minuten das Videospiel Super Mario 64 spielen [25]. Im Vergleich zu einer Kontrollgruppe zeigten sich bei den Teilnehmern der Videospielgruppe Größenzunahmen im rechten Hippocampus und dIPFC sowie in Bereichen des Kleinhirns. Die Autoren ziehen weitreichende Konsequenzen aus ihren Befunden, spielen doch der Hippocampus und der dIPFC bei einer Reihe von psychiatrischen Erkrankungen wahrscheinlich eine Rolle: „Das vorgestellte Videospiel-Training könnte daher verwendet werden, um die bekannten Risikofaktoren für psychische Erkrankungen wie ein kleinerer Hippocampus und ein geringeres Volumen des präfrontalen Kortex beispielsweise bei posttraumatischer Belastungsstörung, Schizophrenie und neurodegenerativen Störungen entgegen zu wirken“ ([24], Übersetzung durch den Autor). ${ }^{15}$

15 „The presented video game training could therefore be used to counteract known risk factors for mental disease such as smaller hippocampus and prefrontal cortex volume in, for example, post-traumatic stress disorder, schizophrenia and neurodegenerative disease.“

\title{
Max-Planck-Gesellschaft
}

\author{
Warum Videospielen gut fürs Gehirn sein kann \\ Hirnregionen lassen sich gezielt trainieren \\ 30. OKTOBER 2013 \\ Gehirn Psychologie \\ Videospielen vergrößert Hirnbereiche, die für räumliche Orientierung, \\ Gedächtnisbildung, strategisches Denken sowie Feinmotorik bedeutsam sind. Das zeigt \\ eine aktuelle Studie des Max-Planck-Instituts für Bildungsforschung und der \\ Psychiatrischen Universitätsklinik der Charité im St. Hedwig-Krankenhaus. Die positiven \\ Effekte von Videospielen könnten auch bei der Therapie psychischer Störungen zum \\ Tragen kommen.
}

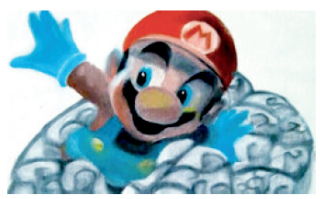

Gute Nachrichten für alle Gamer! Videospielen kann bestimmte Gehirnregionen, z.B. für räumliche Orientierung, Gedächtnisbildung, strategisches Denken, gezielt trainieren.

- Abb.2 Facsimile (Screenshot) vom Beginn der Pressemitteilung der Max-Planck-Gesellschaft vom 30.10.2013 (https://www.mpg.de/7589369/Videospiele-Gehirn; abgerufen am 17.2.2019). Die vermeintliche Erkenntnis, dass Computerspiele die Gehirnentwicklung fördern, war der Max-Planck-Gesellschaft eine eigene Pressemitteilung wert. Herr Planck würde sich im Grabe herumdrehen, wenn er davon erführe, dass mit seinem Namen hier PR für Unterhaltungssoftware betrieben wird, die jungen Menschen nachweislich sehr schadet.

Ein Blick auf $>$ Abb. 1 zeigt jedoch, dass der präfrontale Kortex in dem Altersbereich, in dem sich die Probanden befanden normalerweise noch abnimmt, das „Training“ also die normale Entwicklung beeinträchtigt hat. Die Interpretation der Größenzunahme des dIPFC steht damit im klaren Widerspruch zu den bekannten Ergebnissen aus der Biomarkerforschung zu Risikofaktoren von psychischen Störungen. Dies sei hier klar hervorgehoben: Würde man den Gedanken der Autorin konsequent praktisch folgen, ergäbe sich der Ratschlag, der Schizophrenie oder der Demenz mittels Computerspiele vorzubeugen. Dies ist in höchstem Maße unverantwortlich, denn wir wissen um die negativen Auswirkungen von Computerspielen auf die körperliche und geistige Gesundheit der Menschen.

Dennoch erreichte diese Studie große Popularität ( $\triangleright$ Abb. 2) und vor allem weite mediale Aufmerksamkeit: Wissenschaftler einer sehr renommierten deutschen Wissenschaftlichen Fachgesellschaft hatten festgestellt, dass das Spielen von Videobzw. Computerspielen dem Gehirn guttut. Gamer brauchten von nun an kein schlechtes Gewissen mehr haben. Eltern, die ihren
Kindern eine Playstation schenken, auch nicht. Mehr noch: Computerspielen könne präventiv wirken und Risikofaktoren psychiatrischer Erkrankungen entgegenwirken.

Nicht nur die Dicke des Kortex wurde fehlinterpretiert, sondern dessen Aktivierung in funktionellen Studien auch: Seit dem schon sehr alten Befund, dass nach motorischem Training die Aktivierung der motorischen Gehirnrinde während motorischer Aufgaben abnimmt, lassen sich sowohl Zunahme als auch Abnahme der Aktivierung irgendeines kortikalen Areals im Sinne einer Funktionsverbesserung interpretieren: Mehr Aktivität ist besser, weil mehr eben besser ist, und weniger Aktivität ist auch besser, weil das ein klares Zeichen von effizienterer Nutzung darstellt.

Tatsächlich findet man beide Interpretationen in der Literatur zu Videospielen, jeweils so, wie man es braucht, um deren positive Auswirkungen festzustellen: In einer Arbeit aus dem Jahr 2018 an 16 Spielern und 16 Nicht-Spielern wird vorgemacht, wie es geht (die Letztautorin ist Frau Bavelier): "Während des Hinweisreizes rekrutierten die Action-Videospiel-Spieler 
das Top-down-Aufmerksamkeitsnetzwerk in geringerem Maße als die Nicht-Videospiel-Spieler, was auf eine größere Effizienz dieses Netzwerks bei Action-Videospiel-Spielern hinweist. Während der Bearbeitung des Zielreizes korreliert die Rekrutierung von Top-down-Arealen mit größerer Schwierigkeit der Verarbeitung, aber nur bei den Nicht-Videospiel-Spielern. Bei den Action-Videospiel-Spielern wurde dieser Effekt nicht beobachtet, sondern eine stärkere Aktivierung beider Netzwerke [Bottom-up und Top-down] “ ([10], S.e1). ${ }^{16}$ Bei Videospielern wurden also Aktivierungen und Deaktivierungen gefunden. In ihrer Conclusio sprechen die Autoren dann von einer „funktionalen Rekonfiguration von Top-down- und Bottom-up- Aufmerksamkeitsnetzwerken “ bei den Spielern von Action Video Games. Und sie finden das ganz toll. Was immer es bedeuten mag.

Man sollte sich weiterhin über eine methodische Schwäche aller Studien im Klaren sein, in denen Gamer mit Nicht-Gamern verglichen: Sämtliche Effekte könnten darauf beruhen, dass Menschen, die gerne schnell reagieren, gerne solche Spiele spielen. Man spricht von Selbstselektion („self selection") ein wichtiger und häufiger in Studien zu diskutierendem Effekt. Das hindert die Autoren der gerade genannten Studie jedoch nicht, das Gleiche nochmals im Journal of Cognitive Neuroscience zu publizieren [10].

Ungeachtet der angeführten Studien zu den nicht vorhandenen positiven Auswirkungen von Computer- und Videospielen auf Aufmerksamkeitsprozesse und den bekannten negativen Auswirkungen beginnen sie ihre Arbeit wie folgt (die Letztautorin dieser Arbeit ist wieder Frau Bavelier): „Action Video Game Spieler übertreffen Menschen, die keine Action Video Games spielen, in einer Reihe von Wahrnehmungs- und Aufmerksamkeits-Aufgaben“"([10], S. 377,

16 "During the cue period, action video game players (AVGPs) recruited the top-down network less than nonvideo game players (NVGPs), [...] in line with a greater efficiency of that network in AVGPs. During target processing, as previously shown, recruitment of top-down areas correlated with greater processing difficulties, but only in NVGPs. AVGPs showed no such effect, but rather greater activation across the two networks."

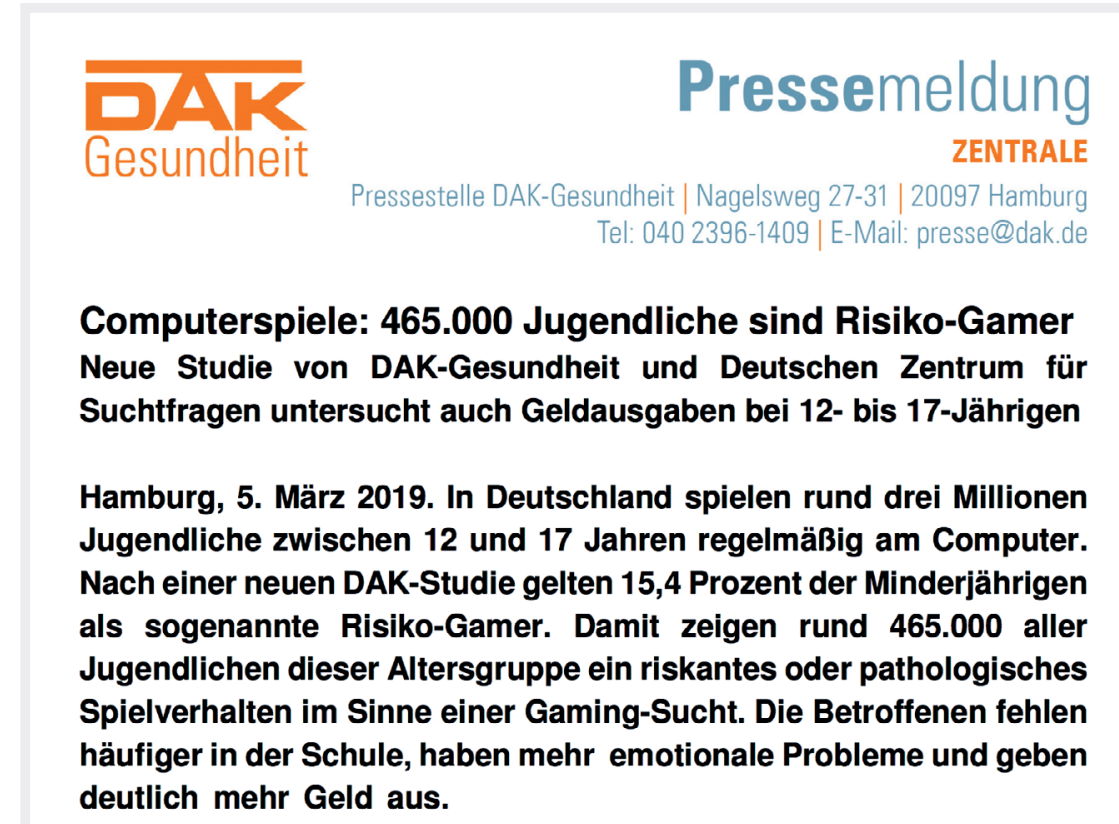

Abb. 3 Facsimile (Screenshot, geringgradig nachbearbeitet) vom Anfang der Pressemitteilung der DAK vom 5.3.2019 zur Häufigkeit Computerspielsucht in Deutschland.

Übersetzung durch den Autor). ${ }^{17}$ Gefunden wurden dann beim Vergleich von 14 Spielern und 14 Nicht-Spielern Verstärkungen und Abschwächungen in unterschiedlichen Komponenten ereigniskorrelierter Potenziale (EKP), die hier nicht weiter betrachtet werden sollen, da zum einen Signaländerungen immer beide Interpretationen zulassen und ein Mehr oder Weniger immer Ausdruck von Besser oder Schlechter und umgekehrt sein kann. Zum andern besteht ein weiterer möglicher Einfluss im Sinne der Selbstselektion.

\section{Diskussion}

Computerspiele sind zunächst einmal kommerziell angebotene, nachweislich zur Erzeugung von Sucht programmierte Softwarepakete, mit denen $80 \%$ unserer jungen Leute ihre Zeit vertreiben. Nach neuesten Angaben sind, wie eingangs bereits erwähnt, in Deutschland 465000 junge Menschen von Computerspielsucht betroffen (DAK 5.3.2019; - Abb.3), in China waren

17 „Action video game players (AVGPs) outperform non-action video game players (NAVGPs) on a range of perceptual and attentional tasks.“ es vor 10 Jahren schon 24 Millionen (nach [54]; neueste Zahlen lauten ähnlich [27]).

Die Zusammenhänge zwischen dem Spielen von Computerspielen und Aufmerksamkeitsstörungen (ADHD), Ängsten (vor allem soziale Phobie), Depression (einschließlich Suizidalität) und anderen stoffgebundenen Süchten (Alkohol, Nikotin, illegale Drogen $)^{18}$ ist bekannt und gut dokumentiert [12, 33, 44, 56].

Auch die Probleme im somatischen Bereich - Adipositas und Haltungsschäden sowie längerfristig Diabetes und Hypertonie mit dem langfristigen Risiko erhöhter kardiovaskulärer Komplikationen wie Schlaganfälle und Herzinfarkte - durch Computerspiele sind schwerwiegend und verursachen erhebliche Kosten im Gesundheitswesen. Seit die Internet- und Computersucht durch die WHO als Krankheit anerkannt ist, wird auch zunehmend deutlich, dass die Zahl der Behandlungsplätze - auf dem deutschen Suchtkongress im September 2018 war von 200 die Rede - in krassem Missverhältnis zur Zahl der Betroffenen (über 400000) steht.

18

8 So werden das Rauchen sowie andere Drogen oft in Computerspielen indirekt beworben $[11,30]$. 
In der Literatur zu den vermeintlich positiven Auswirkungen von Computerspielen auf Geist und Gehirn werden diese bekannten Risiken und Nebenwirkungen systematisch ausgeblendet. So entstand eine vermeintlich „wissenschaftliche“ Literatur, die beständig zitiert wird, wenn es um die Frage der gesundheitlichen Schäden geht. Es wird damit von ganz offizieller Seite „Entwarnung“ gegeben. Damit wird Wissenschaft auf verantwortungslose Weise missbraucht ( $\triangleright$ Abb. 2).

Wie weit diese „Industrie“ zur Herstellung vermeintlich wissenschaftlicher Arbeiten zu den positiven Auswirkungen von Computerspielen geht, mag das folgende Beispiel andeuten:

Im Jahr 2017 erschien im respektablen Fachblatt Current Directions in Psychological Science eine Arbeit mit dem Titel „Video Games and Hippocampus-Dependent Learning" [51]. Die Arbeit handelt davon, wie man das vorhandene Wissen um die Funktionen des Hippocampus nutzen könnte, um Video- und Computerspiele im Hinblick auf ihren Trainingseffekt zu verbessern. Es werden keine Daten erhoben und es wird nichts untersucht. Vielmehr wird vorgeschlagen, in elektronischen Spielen auf das - simulierte - Satelitennavigationssystem (GPS) zu verzichten, weil dadurch die Ausbildung hippocampaler Landkarten gefördert werden könnte. Die ganze Arbeit ist also eine hypothetische Erörterung - und sonst nichts! Aber ihr Titel suggeriert einen Zusammenhang zwischen Video-Games, Lernen und einem Gehirnbereich, den man aus der Literatur zu Lernprozessen seit den Zeiten des berühmten Patienten H. M. schon einmal gehört hat: dem Hippocampus.

Fassen wir zusammen: die Literatur zu den vermeintlichen positiven Auswirkungen von Video- und Computerspielen ist so etwas wie organisierte Schaumschlägerei: Durch schlecht gemachte Studien und vor allem auf den Kopf gestellte Interpretationen werden positive Auswirkungen suggeriert, die sich bei genauem Hinsehen in Luft auflösen. Die bekannten, gut dokumentierten und für das Gesundheitswesen kostenrelevanten Schäden werden in dieser Literatur systematisch verschwiegen. Produziert wird dadurch ein ganzes Literaturgenre, das sich „Wissenschaft" nennt, aber keine ist (man nennt so etwas auch „Etikettenschwindel“), und dass es nur geben kann, wenn eine sehr starke Lobby dahintersteht, um deren finanzielle Interessen es geht.

Computerspiele wurden in Anlehnung an die Begriffe Hardware und Software auch als Exploitationware, d. h. als Instrument der Ausbeutung, bezeichnet [4]. Ausgebeutet werden Kinder und Jugendliche, deren Schutz zu den wichtigsten Aufgaben der erwachsenen Bevölkerung gehören sollte. Dass das Gegenteil tatsächlich geschieht - wie der Koalitionsvertrag der gegenwärtigen Regierung deutlicher nicht zeigen könnte - und das Computerspielen sogar für gemeinnützig erklärt werden soll, also für steuerlich begünstigt und förderungswürdig, zeigt an, wie gut die Strategie der Lobby funktioniert. Es wird Zeit, dass Vertreter von Wissenschaft und Medizin - um die Glaubwürdigkeit von beidem geht es - hier klarer Flagge zeigen. Wer es nicht tut, handelt verantwortungslos.

\section{Interessenkonflikt}

Es liegen keine Interessenkonflite vor.

Korrespondenzadresse

Prof. Dr. Dr. Manfred Spitzer

Universität Ulm

Abteilung für Psychiatrie

Leimgrubenweg 12-14

89075 Ulm

\section{Literatur}

[1] Al-Thaqib A et al. Brain training games enhance cognitive function in healthy subjects. Med Sci Monit Basic Res 2018; 24 63-69.

[2] Ballesteros $\mathrm{S}$ et al. Effects of video game training on measures of selective attention and working memory in older adults: Results from a randomized controlled trial. Front Aging Neurosci 2017; 9: 354.

[3] Bitkom. Gaming hat sich in allen Altersgruppen etabliert. 29.7.2015 (https://www. bitkom.org/Presse/Presseinformation/Gaming-hat-sich-in-allen-Altersgruppen-etabliert.html; abgerufen am 3.3.2019)
[4] Bogost I. Gamification is Bullshit‘. The Atlantic, August 2011 (http://www.theatlantic.com/technology/archive/2011/08/ gamification-is-bullshit/243338/; abgerufen am 5.3.2016)

[5] Botdorf M, Riggins T. When less is more: Thinner fronto-parietal cortices are associated with better forward digit span performance during early childhood. Neuropsychologia 2018; 121: 11-18.

[6] Chaddock-Heyman L et al. Physical activity increases white matter microstructure in children. Front. Neurosci 2018; 12: 950.

[7] Chaddock-Heyman L et al. The role of aerobic fitness in cortical thickness and mathematics achievement in preadolescent children. PLoS ONE 2015; 10(8): e0134115.

[8] DAK (Deutsche Angestelltenkrankenkasse). Computerspiele: 465.000 Jugendliche sind Risiko-Gamer. Pressemitteilung vom 5.3.2019 (https://www.dak.de/dak/ download/pressemitteilung-computerspielsucht-2053898.pdf; abgerufen am 25.3.2019)

[9] Föcker ], Cole D2, Beer AL I, Bavelier D. Neural bases of enhanced attentional control: Lessons from action video game players. Brain and Behavior 2018; 8: e010191.

[10] Föcker ] et al. Neural correlates of enhanced visual attentional control in action video game players: An event-related potentia study. Journal of Cognitive Neuroscience 2019; 31: 377-389.

[11] Forsyth SR, Malone RE. Smoking in video games: A systematic review. Nicotine Tob Res 2016; 18(6): 1390-8.

[12] Gentile DA et al. Video game playing, attention problems, and impulsiveness: Evidence of bidirectional causality. Psychology of Popular Media Culture 2012; 1: 62-70.

[13] Giedd JN et al. Brain development during childhood and adolescence: a longitudinal MRI study. Nat Neurosci 1999; 2: 861-863.

[14] Giedd JN et al. Puberty-related influences on brain development. Mol Cell Endocrinol 2006; 254-255: 154-162.

[15] Giedd JN et al. Anatomical brain magnetic resonance imaging of typically developing children and adolescents. J Am Acad Child Adolesc Psychiatry 2009; 48: 465-470.

[16] Gogtay N et al. Dynamic mapping of human cortical development during childhood through early adulthood. PNAS 2004; 101: 8174-8179.

[17] Green CS, Bavelier D. Action video game modifies visual selective attention. Nature 2003; 423: 534-535.

[18] Have M et al. Classroom-based physical activity improves children's math achievement - A randomized controlled trial. PLoS One 2018; 13(12): e0208787.

[19] Hsieh SS et al. The subject-dependent, cumulative, and recency association of aerobic fitness with academic performance 
in Taiwanese junior high school students. BMC Pediatr 2019; 19(1): 25.

[20] Kable JW et al. No effect of commercial cognitive training on brain activity, choice behavior, or cognitive performance. J Neurosci 2017; 37: 7390-7402.

[21] Karbach ], Verhaeghen P. Making working memory work: A meta-analysis of executive-control and working memory training in older adults. Psychological Science 2014; 25: 2027-2037.

[22] Katz B, Shah P, Meyer DE. How to play 20 questions with nature and lose: Reflections on 100 years of brain-training research. PNAS 2018; 115: 9897-9904.

[23] Kietglaiwansiri T, Chonchaiya W. Pattern of video game use in children with attention-deficit-hyperactivity disorder and typical development. Pediatr Int 2018; 60: 523-528.

[24] Kühn S et al. Playing Super Mario induces structural brain plasticity: Gray matter changes resulting from training with a commercial video game. Molecular Psychiatry 2014; 19: 265-271.

[25] Kühn S et al. Positive association of video game playing with left frontal cortical thickness in adolescents. PLoS ONE 2014; 9(3): e91506.

[26] Lee D. Top video games depict unrealistic drugs use says study (http://www.bbc.com/ news/technology-42808573)

[27] Li L et al. Prevalence of Internet addiction disorder in Chinese university students: A comprehensive meta-analysis of observational studies. J Behav Addict 2018; 7: 610-623.

[28] Mathews CL, Morrell HER, Molle JE. Video game addiction, ADHD symptomatology, and video game reinforcement. Am J Drug Alcohol Abuse 2019; 45: 67-76.

[29] Melby-Lervåg M, Hulme C. Is working memory training effective? A meta-analytic review. Developmental Psychology 2013; 49: 270-291.

[30] Munafò M. Tobacco marketing by stealth. Nicotine Tob Res 2016; 18(6): 1389.

[31] Owen AM et al. Putting brain training to the test. Nature 2010; 465: 775-778.

[32] Pallavicini F et al. Video games for wellbeing: A systematic review on the application of computer games for cognitive and emotional Ttaining in the adult population. Front Psychol 2018; 9: 2127.

[33] Peterka-Bonetta J et al. The relationship between internet use disorder, depression and burnout among Chinese and German college students. Addict Behav 2019; 89: 188-199.

[34] Peterson A, Fung B. FTC: 'Brain training' brand Lumosity didn't have the research to back up its claims. Washington Post 5.1.2016 (https://www.washingtonpost. com/news/the-switch/wp/2016/01/05/ftc- brain-training-brand-lumosity-didnt-havethe-research-to-back-up-its-claims/?utm_ term=.be4d1 ce 38796

[35] Ra CK et al. Association of digital media use with subsequent symptoms of attention-deficit/hyperactivity disorder among adolescents. JAMA 2018; 320: 255-263.

[36] Riggins T et al. Protracted hippocampal development is associated with age-related improvements in memory during early childhood. Neuroimage 2018; 174 : 127-137.

[37] Rossignoli-Palomeque T et al. Brain training in children and adolescents: Is it scientifically valid? Front Psychol 2018; 9: 565.

[38] Rumpf H] et al. Including gaming disorder in the ICD-11: The need to do so from a clinical and public health perspective. J Behav Addict 2018; 7: 556-561.

[39] Sala G Gobet F. Cognitive training does not enhance general cognition. TICS 2019; 23: 9-20.

[40] Shaw P et al. Attention-deficit/hyperactivity disorder is characterized by a delay in cortical maturation. PNAS 2007; 104: 19649-19654.

[41] Simons D] et al. Do "Brain-Training" programs work? Psychological Science in the Public Interest 2016; 17: 103-186.

[42] Spitzer M. E-Sport. Nervenheilkunde 2019; 38: 157-168.

[43] Steyvers M, Benjamin AS. The joint contribution of participation and performance to learning functions: Exploring the effects of age in large-scale data sets. Behav Res Methods 2018 Sep 24. doi: 10.3758 s13428-018-1128-2.

[44] Stockdale L, Coyne SM. Video game addiction in emerging adulthood: Cross-sectional evidence of pathology in video game ad dicts as compared to matched healthy controls. J Affect Disord 2018; 225: 265-272.

[45] Straub J et al. Adolescent depression and brain development: Evidence from voxel-based morphometry. J Psychiatry Neurosci Published online Feb. 5, 2019; DOI: 10.1503/jpn.170233.

[46] Tamnes CK et al. Development of the cerebral cortex across adolescence: A multisample study of inter-related longitudinal changes in cortical volume, surface area, and thickness. J Neurosci 2017; 37: 3402-3412.

[47] Thao-Ly TP et al. Electronic gaming characteristics associated with class 3 severe obesity in youth who attend the pediatric weight management programs of the COMPASS Network. Childhood Obesity 15, January 2, 2019; doi.org/10.1089/chi 2018.0156.

[48] Thorpe S, Fize D, Marlot C. Speed of processing in the human visual system. Nature 1996; 381: 520-522.
[49] Unsworth $\mathrm{N}$ et al. Is playing video games related to cognitive abilities? Psychological Science 2015; DOI: 10.1177/0956797615570367.

[50] Watson A et al. Associations between organised sport participation and classroom behaviour outcomes among primary school-aged children. PLoS ONE 2019; 14(1): e0209354.

[51] West GL, Konishi K, Bohbot VD. Video games and hippocampus-dependent learning. Current Directions in Psychological Science 2017; 26: 152-158.

[52] Wikipedia. Federal Trade Commission (FTC) (https://de.wikipedia.org/wiki/Federal_Trade_Commission; abgerufen am 2.3.2019).

[53] Wikipedia. Lumosity. (https://en.wikipedia. org/wiki/Lumosity

[54] Yuan K et al. Cortical thickness abnormalities in late adolescence with online gaming addiction. PLoS ONE 2013; 8(1): e53055.

[55] Zheng F et al. Association between mobile phone use and inattention in 7202 Chinese adolescents: A population based cross-sectional study. BMC Public Health 2014; 14: 1022-1028.

[56] González-Bueso V et al. Association between internet gaming disorder or pathological video-game use and comorbid psychopathology: A comprehensive review. Int J Environ Res Public Health 2018; 15 : 668.

[57] Dye MWG, Green CS, Bavelier D. The development of attention skills in action video game players. Neuropsychologia 2009; 47: 1780-1789.

[58] Bavelier D, Green CS, Dye MWG. Children, wired: For better and for worse. Neuron 2010; 67: 692-701.

\section{Bibliografie}

DOI https://doi.org/10.1055/a-0847-8627

Nervenheilkunde 2019; 38: 314-323

(c) Georg Thieme Verlag KG Stuttgart - New York ISSN 0722-154 